Robin Crawford

\title{
Laparoscopic management of adnexal masses in adolescent females: a multidisciplinary approach
}

Published online: 12 August 2005

(C) Springer-Verlag Berlin / Heidelberg 2005

\begin{abstract}
Laparoscopic surgery could be considered the gold standard in managing adnexal masses. The advantages of laparoscopic surgery have been proven in the adult population, and thus its use has been promoted for treating children and adolescents. However, although adolescent females should not be treated like children, their management differs from that of adults. We present three cases to illustrate how three adolescent girls had their adnexal masses dealt with laparoscopically. We recommend that hospitals consider a combined approach in the management of large adnexal masses in adolescents by utilising the expertise of the adolescent gynaecologist, gynaecology oncologist, and minimal access surgeon. In certain centres, a particular gynaecologist may possess all the skills required.
\end{abstract}

Keywords Adnexal mass - Adolescent - Laparoscopy

\section{Case report}

Operative laparoscopy has developed dramatically and quickly. Laparoscopic surgery could be considered the gold standard in managing adnexal masses. Its value has been demonstrated in prospective randomised trials [1]. The advantages of laparoscopic surgery have been proven in the adult population, and thus its use has been promoted for treating children and adolescents. A recent paper describes its role in the management of intersex disorders [2].

\footnotetext{
Y. L. Woo $(\bowtie) \cdot J$. Latimer $\cdot$ R. Crawford

Department of Gynaecology Oncology,

Addenbrooke's NHS Trust, Addenbrooke's Hospital,

Box 242, Hills Road, Cambridge CB1 2QQ, UK

E-mail: ylw22@cam.ac.uk

Fax: + 44-1223-217666

J. MacDougall

Department of Obstetrician and Gynaecologist,

Addenbrooke's Hospital,

Cambridge CB1 2QQ, UK
}

The laparoscopic management of an adnexal mass hinges on an accurate assessment of it. Ideally, the management of adnexal mass in adolescent females should be undertaken by gynaecologists with expertise in adolescent gynaecology as well as an understanding of the biology of benign and malignant adnexal masses. However, although adolescent females should not be treated like children, their management differs from that of adults; they pose a different set of problems.

First, the consultation is usually in the presence of parents who are anxious, particularly when dealing with problems that can potentially have an impact on their child's fertility. The discussion of gynaecological problems is frequently a difficult topic for both the parents and the teenager, even when fertility has not been considered.

Second, the pathology in adolescents is different from that in adults. Even within the adolescent age group, the pathology varies depending on the patient's age. In a series of 140 patients, it was found that ovarian malignancy was more common in girls under 15 , with ovarian cysts and benign tumours more likely in older patients [3]. This finding was confirmed in a larger series from the Mayo Clinic [4]. The type of malignancy also has an age distribution; germ cell tumours are most common in the first decade of life, whereas those of epithelial origin occur in older adolescents.

Third, adolescent patients often require different investigations than their adult counterparts. In younger adolescents, transvaginal ultrasound may not be possible due to an intact hymen. Additional tumour markers such as alpha-fetoprotein (AFP), lactate dehydrogenase (LDH), carcinoembryonic antigen (CEA), and human gonadotrophin (HCG) should be requested.

The diagnosis of adnexal mass in the young person requires a thorough history. Specific enquiry into symptoms such as precocious puberty and hirsutism must be undertaken. Ideally, a sexual history should be elicited in a private setting without the presence of the parents. 
The most appropriate radiological test should be utilised to gain the most preoperative information. Ultrasound has remained the mainstay of diagnosis and usually serves as the initial radiological investigation of choice, often allowing detailed characterisation of adnexal masses. When more information is required, the new standard for diagnosis has become magnetic resonance imaging (MRI) [5]. The involvement of a radiologist with an interest in pelvic masses may enhance patient care. In our unit, after discussion with an experienced radiologist and review of the existing radiology, a repeat radiological investigation is often undertaken to look for specific features within the adnexal mass.

The multidisciplinary approach between the adolescent gynaecologist and the gynaecology oncologist in our unit works well for managing adnexal masses. When there is a low risk of malignancy, even very large masses are dealt with laparoscopically. The size of the ovarian cyst is a major factor limiting wider application of laparoscopic management in benign ovarian cysts. When confronted with adnexal masses $>10 \mathrm{~cm}$, most surgeons would choose to perform laparotomy. We have provided three cases to illustrate how three adolescent girls had their adnexal masses dealt with laparoscopically.

\section{Case 1}

A 13-year-old girl presented with a 4-month history of cyclical abdominal pain and an abdominal swelling. She had reached her pubarche and thelarche 6 months prior to presentation. The initial clinical diagnosis elsewhere was that of an imperforate hymen. Ultrasound demonstrated a central cystic swelling. The girl was referred to an adolescent gynaecologist, was examined, and was found to have normal external genitalia and hymenal opening. MRI of the pelvis revealed a left ovarian cyst $7 \mathrm{~cm}$ in size with no solid elements. Her CA 125 was $25 \mathrm{u} / 1$, and all other tumour markers were negative.

After full discussion with the patient and her parents, it was decided that this cystic swelling could be dealt with laparoscopically. This would be undertaken jointly between the adolescent gynaecologist and the gynaecology oncologist.

As with all laparoscopic cases in the gynaecology oncology unit, open laparoscopy was adopted. A 2-cm vertical incision was made in the inferior part of the umbilicus. The subcutaneous tissue and sheath were gently dissected using a pair of scissors. Once through the sheath, using two skin retractors (small Langenbecktype retractors), the sheath was elevated from the peritoneum. The peritoneal cavity was then carefully entered. Once in, the two edges (sheath and peritoneum) were secured with a long length of dissolvable suture, such as Vicryl, using a J-needle, leaving about $20 \mathrm{~cm}$ of suture on both sides of the edges. Using the two lengths of suture, the anterior abdominal wall was elevated prior to inserting the blunt Hassan port. Once the port was in the peritoneal cavity, pneumoperitoneum up to a pressure of $20 \mathrm{mmHg}$ was achieved using $\mathrm{CO}_{2}$ insufflation. Two further $5-\mathrm{cm}$ ports were inserted at both lower quadrants under direct vision.

At operation the girl was found to have a torted left ovarian cyst along with the fallopian tube. The omentum was also adherent to the torted ovary. The right ovary and fallopian tube were normal. It was decided that the best option was to perform a left salpingooophorectomy. Peritoneal washings were taken. Using a Gyrus Medical bipolar cutting forceps 3005PK, the infundibulopelvic ligament was diathermied and then divided, and the left adnexa was detached. A tissue retrieval bag (Tissue Retrieval System, EMP 100-INS, Espiner Medical Products, UK) was then introduced via the umbilical port. With two graspers, the ovary and tube were placed inside the bag. The bag was subsequently removed through the incision in the umbilicus. In this case, the bag could not be removed via the umbilical incision with the contents in it; the contents had to be slowly removed using a polyp forceps to decompress the bag. The bag was then pulled through the small incision.

\section{Case 2}

A 17-year-old girl with cystic fibrosis was found to have an $18 \times 14-\mathrm{cm}$ simple right ovarian cyst during the course of investigations for constipation. For further management she was referred to the gynaecologist running the adolescent clinic. Her CA 125 was $46 \mathrm{u} / \mathrm{l}$, and other tumour markers were negative. There were concerns that a laparotomy would compromise her postoperative recovery as it could potentially exacerbate her already compromised lung function. Understandably, there were also questions surrounding the issue of future fertility. After a multidisciplinary meeting between the adolescent gynaecologist, the chest physicians, the anaesthetist, and the gynaecology oncologist, it was decided that the best option would be to deal with the cyst laparoscopically.

The patient was covered by antibiotics pre- and intraoperatively to reduce the risk of a lung infection. Laparoscopy was undertaken using the open technique as described in case 1 . An $18-\mathrm{cm}$ cyst was found to be attached to the ovary, with healthy ovarian tissue separate from it. The fallopian tube was, unfortunately, stretched around the cyst. Peritoneal washings were performed. The cyst was decompressed and dissected from the remaining healthy ovarian tissue. The deflated tissue as well as the fallopian tube were then removed by use of a tissue retrieval bag as described in case 1 .

\section{Case 3}

A 13-year-old girl presented with a 2-month history of lower abdominal swelling. She had no other associated 
symptoms. She was premenarchal. Ultrasound revealed an $18-\mathrm{cm}$ ovarian cyst, which was smooth and had no solid elements. All the tumour markers including CA125 were normal.

Open laparoscopy was performed, and peritoneal washings were taken. The impression was that of a dermoid cyst. By use of a 5-cm suprapubic incision, the cyst was punctured and drained. The cyst was removed, and the ovary was reconstituted extraperitoneally. There was minimal spillage of cyst contents into the peritoneal cavity. The ovary was then placed back into the pelvis (Table 1).

\section{Discussion}

In general, the above patients would have had their adnexal masses dealt with by laparotomy. This is reasonable when resources and expertise are limited. However, in centres where there are experienced laparoscopic surgeons, gynaecology oncologists, and reproductive medicine specialists, a multidisciplinary effort in grading the risks of malignancy and deciding the surgical approach would be ideal. Such a meeting should take place with the results of the relevant investigations (tumour markers and radiology). Ideally, the multidisciplinary meeting should be initiated by the person who had undertaken a thorough consultation with the patient. As the team's experience broadens, confidence in management decisions should increase. If there is a low suspicion of a malignancy, even very large cysts (above the level of the umbilicus) can be approached laparoscopically, as demonstrated in cases 2 and 3 . A recent case report describes the laparoscopic removal of an 8$\mathrm{cm}$ dermoid cyst [6]. In case 3 , using a 5 -cm suprapubic incision, an 18-cm dermoid cyst was removed successfully. In all three cases, the postoperative stay was minimal.

The open procedure allows direct entry and achievement of pneumoperitoneum without a Verres needle. In our experience, there is minimal risk of puncturing the cyst using such an approach. It allows visualisation of the peritoneal cavity and any large masses prior to inserting a trocar. Subsequent ports are also inserted under direct vision, therefore with minimal risk of puncturing the cystic mass or other organs. In all such cases, it is always good practice to take peritoneal washings for cytology prior to extensive manipulation. It is also important to ensure that the cyst contents do not spill into the peritoneal cavity. In general, a tissue retrieval bag, which comes in different sizes, is extremely useful for removing the mass without spillage. This should be the preferred method. If the cyst is too big to be inserted into a bag for decompression and removal, extraperitoneal decompression via a small laparotomy incision followed by removal or replacement, as in case 3 , should achieve the same outcome.

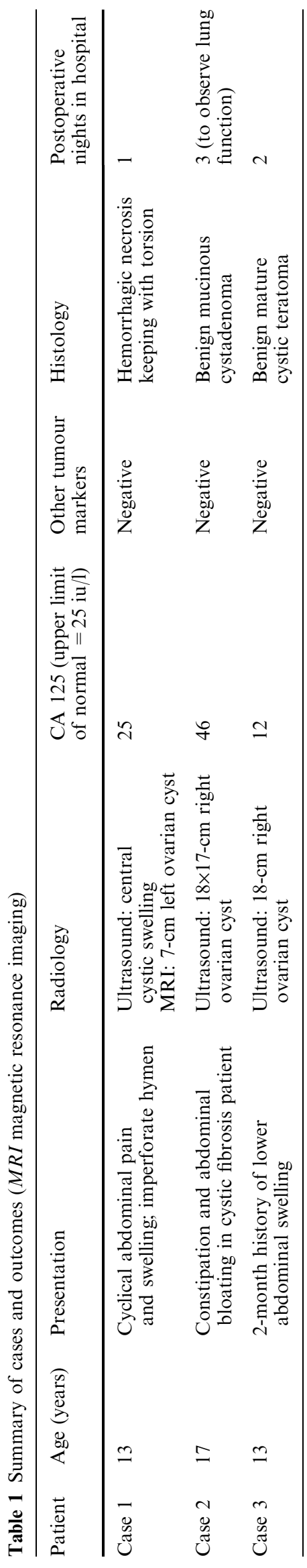


With experience, dealing with large adnexal masses involves a similar operating time as open laparotomy. The benefits gained postoperatively from a laparoscopic procedure far outweigh any marginal time saved by performing a laparotomy.

We therefore recommend that hospitals consider a combined approach in the management of large adnexal masses in adolescents by utilising the expertise of the adolescent gynaecologist, gynaecology oncologist, and minimal access surgeon. In certain centres, a particular gynaecologist may possess all the skills required.

\section{References}

1. Canis MRB, Houlle C, Botchorishvili R, Jardon K, Safi A, Wattiez A, Mage G, Luc Pouly J, Bruhat M (2002) Laparoscopic management of adnexal masses: a gold standard? Curr Opin Obstet Gynaecol 14:423-428
2. Esegbona G, Cutner A, Cuckow P, Creighton S (2003) Laparoscopic gonadectomy in paediatric and adolescent girls with intersex disorders. BJOG 110(2):210-212

3. Templeman CFM, Blinchevsky A, Hertweck P (2000) Noninflammatory ovarian masses in girls and young women. Obstet Gynecol 96(2):229-233

4. Van Winter JSP, Podratz K (1994) Surgically treated adnexal masses in infancy, childhood, and adolescence. Am J Obstet Gynecol 170(6):1780-1789

5. Logsdon VK (2001) Common problems in pediatric and adolescent gynaecological surgery. Curr Opin Obstet Gynaecol $13: 453-458$

6. Sonmezer MGM, Gengiz B, Ortac F (2002) Laparoscopic extracorporeal cystectomy in the management of large dermoid cysts. Gynaecol Endosc 11:201-204 